\title{
On the Use of a Direct Radiative Transfer Equation Solver for Path Loss Calculation in Underwater Optical Wireless Channels
}

\author{
Changping Li, Ki-Hong Park, Member, IEEE, and Mohamed-Slim Alouini, Fellow, IEEE
}

\begin{abstract}
In this letter, we propose a fast numerical solution for the steady state radiative transfer equation based on the approach in [1] in order to calculate the optical path loss of light propagation suffering from attenuation due to the absorption and scattering in various water types. We apply an optimal non-uniform method to discretize the angular space and an upwind type finite difference method to discretize the spatial space. A Gauss-Seidel iterative method is then applied to solve the fully discretized system of linear equations. Finally, we extend the resulting radiance in 2 -dimensional to 3 -dimensional by the azimuthal symmetric assumption to compute the received optical power under the given receiver aperture and field of view. The accuracy and efficiency of the proposed scheme are validated by uniform RTE solver and Monte Carlo simulations.
\end{abstract}

Index Terms-Radiative transfer equation, underwater optical path loss, finite difference method, Gauss-Seidel iteration.

\section{INTRODUCTION}

W ITH the increase of human activity in ocean exploration, the traditional acoustic communication is inappropriate for high speed communications underwater. Thanks to much higher spectral efficiency, optical wireless communication has been attracted as an emerging technology. Due to two mechanisms corrupting light propagation, absorption and scattering, the underwater is much more complex and challenging environment than free space. It is reported in [2] that light beam suffers from much higher (about 100-1000 times) attenuation in water than air. Therefore, one of the main targets in underwater optical wireless communication (UOWC) channel modeling is to evaluate the overall path loss for calculating link budgets and signal-to-noise ratio.

The Beer's law, the simple exponential attenuation model, is typically applied to calculate the optical path loss owing to its simplicity [3]. However, it is assumed that all the scattered photons are lost even though, in fact, some of the scattered photons can still be captured by the receiver after multiple scattering. By doing so, it can severely underestimate the received power, especially in the scattering dominant regime. On the other hand, the radiative transfer equation (RTE) is a general theoretical model which takes the multiple scattering into account. It fully describes the behavior of energy conservation

C. Li, K.-H. Park, and M.-S. Alouini are with the Computer, Electrical, and Mathematical Sciences and Engineering (CEMSE) Division, King Abdullah University of Science and Technology (KAUST), Thuwal, Makkah Province, Saudi Arabia. (E-mail: lichangping1012@hotmail.com; \{kihong.park, slim.alouini\}@kaust.edu.sa)

This work was supported by a Red Sea Research Center CCF grant from the Office of Sponsored Research at KAUST. for light propagating through an absorbing, scattering and emitting medium. However, the RTE is an integro-differential equation involving several independent variables [4] which cannot analytically find a general solution but numerically.

In view of this, most of the researchers focus on two basic approaches to develop numerical RTE solvers: probabilistic and deterministic methods. The Monte Carlo (MC) simulation is a typical probabilistic approach. It finds a solution by sending and tracking large numbers of photons individually through a water body. This method is intuitive, easy to program, and accurate. However it has drawbacks: i) it is not suitable for the simulation of a point source and a point detector; ii) it cannot address wave phenomena; and iii) it tends to be very time consuming as millions (or billions) of photons are required to accurately simulate a real-world situation [5], [6].

As an alternative, the deterministic numerical approach is highly mathematical but computationally efficient. The conventional discrete ordinate method (DOM) [7] expands the integral term in RTE by the summation of orthogonal bases, which is not local in the angular space. Therefore it can not work well with the highly forward peaked volume scattering functions (VSF) which is the case for ocean water [8]. Recently, a finite element based direct angular discretization scheme is proposed in [1]. Since the integral term in RTE is discretized directly in the angular space, it can capture the characteristic of the scattering type well.

In this letter, we develop a fast direct numerical RTE solver to compute the received power of UOWC systems. Unlike uniform descretization of the angular space as in [1], we propose an optimal non-uniform angular discretization scheme in consideration of the strong forward scattering characteristic of ocean waters to further accelerate the computation process. After angular discretization, an upwind type finite difference method is applied to discrete spatial space. Finally, to solve the fully discretized large system of linear equations, we adopt the matrix free Gauss-Seidel iterative method. Simulation results show that the proposed scheme computes the received power with an accuracy comparable to MC simulations and the uniform solver, while further reducing the computational time.

\section{Proposed RTE Solver}

We modify the general time dependent 3-dimensional (3D) RTE in [4] to the steady state 2-dimensional (2D) RTE as:

$\vec{n} \cdot \nabla L(\vec{r}, \vec{n})=-c L(\vec{r}, \vec{n})+\int_{2 \pi} \beta\left(\vec{n}, \vec{n}^{\prime}\right) L\left(\vec{r}, \vec{n}^{\prime}\right) d \vec{n}^{\prime}+E(\vec{r}, \vec{n})$, 
where we note that all the quantities in (1) are wavelength dependent. In this letter, we omit the wavelength for brevity by assuming that single wavelength is used (blue or green). In (1), $c=a+b\left(m^{-1}\right)$ is the attenuation coefficient which is the summation of the absorption coefficient $a$ and scattering coefficient $b, \nabla$ is the divergence operator. $L(\vec{r}, \vec{n})$ is the optical radiance at position $\vec{r}$ propagating towards direction $\vec{n}$, with unit of $W m^{-2} s r^{-1}, E(\vec{r}, \vec{n})$ is the source radiance, and $\beta\left(\vec{n}, \vec{n}^{\prime}\right)$ is the VSF which is related to the scattering phase function $\tilde{\beta}\left(\vec{n}, \vec{n}^{\prime}\right)$ as

$$
\beta\left(\vec{n}, \vec{n}^{\prime}\right)=b \tilde{\beta}(\theta),
$$

where $\theta$ is defined as the scattering angle between $\vec{n}$ and $\vec{n}^{\prime}$, i.e., $\vec{n} \cdot \vec{n}^{\prime}=\cos \theta$. The phase function describes the angular distribution of the scattered photons. In this letter, we apply the 2D Henyey-Greenstein (H-G) phase function [1], [5]

$$
\tilde{\beta}(\theta)=\frac{1-g^{2}}{2 \pi\left(1+g^{2}-2 g \cos \theta\right)},
$$

where $g$ is the asymmetry parameter with $g \in[0,1]$ which decides the scattering type. For instance, isotropic scattering dominates at $g=0$, while $g$ is close to 1 in presence of peaked scattering. As an example, ocean waters are strong forward scattering media with $g$ values in the range of 0.8 to 0.95 [5], which is potentially beneficial for the communication link.

To solve the integro-differential RTE in (1) numerically, we firstly discretize both angular and spatial variables and then solve the fully discretized large system of linear equations by a Gauss-Seidel iteration method. It is worth to mention that although we assume a homogenous water environment in this letter, the proposed scheme can be applied to a heterogeneous case by simply using averaged values of the coefficient $a, b$ and $c$.

\section{A. Non-uniform Angular Discretization}

The uniform angular discretization in [1] works optimum for isotropic scattering $(g=0)$. However, ocean waters are highly forward scattering media. It has been proved that the $\mathrm{H}$ $\mathrm{G}$ phase function in (3) with asymmetry parameter $g=0.924$ is a good approximation for the angular distribution of the scattered photons in most water types [6]. In view of this, we take advantage of this inherent quality to accelerate the calculation by a non-uniform angular discretization. The basic principle is that we adjust the discretization to the shape of the $\mathrm{H}-\mathrm{G}$ phase function, setting a finer partition in regions where the phase function possesses large value and vice versa. In the $2 \mathrm{D}$ case, the angular variable ranges in $[0,2 \pi)$. As shown in Fig. 1, we discretize the angular space into $K$ directions unequally with the angle interval $\Delta \theta_{k}$. We note that the angular discretization in $(0, \pi)$ and $(\pi, 2 \pi)$ are symmetric in regard to the forward direction. For a given value of $K$, let $N=\frac{K}{2}+1$. We apply Lloyd-Max algorithm [9] to achieve the optimal discrete angles $\left\{\Theta_{k}\right\}$ which minimizes the mean squared error (MSE)

$$
M S E=\sum_{k=1}^{N} \int_{t_{k-1}}^{t_{k}}\left(\theta-\Theta_{k}\right)^{2} \tilde{\beta}(\theta) d \theta,
$$

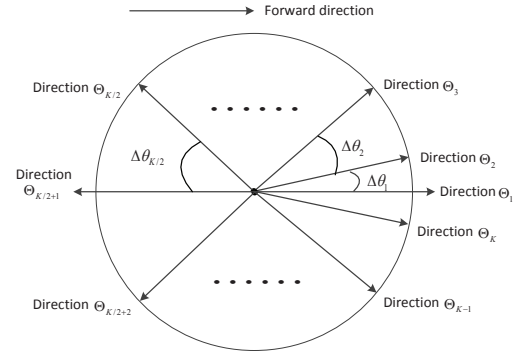

Fig. 1. Direct non-uniform angular discretization.

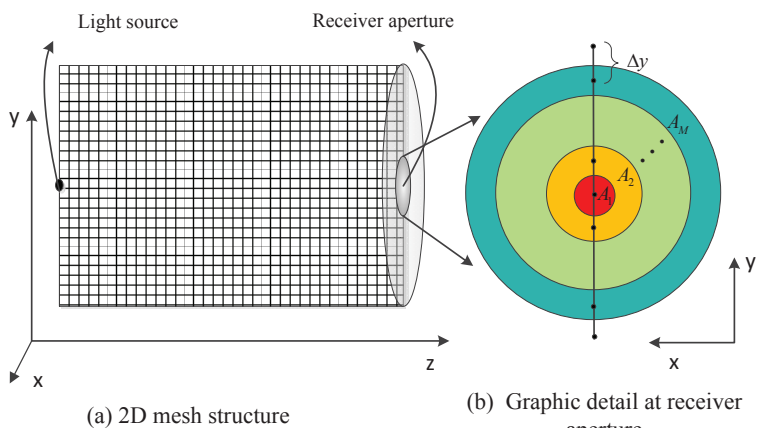

(a) 2D mesh structure aperture

Fig. 2. The overall system configuration.

where $\left\{t_{k}\right\}$ are the decision thresholds. We note that LloydMax algorithm converges to local optima generally which does not guarantee the global optimal solution. Fortunately, in this letter, the phase function in (3) is monotonic within $(0, \pi)$, therefore, the local optimal solution is exactly the global one. To minimize the MSE two conditions are necessary:

$$
\begin{aligned}
\Theta_{k} & =\frac{\int_{t_{k-1}}^{t_{k}} \theta \tilde{\beta}(\theta) d \theta}{\int_{t_{k-1}}^{t_{k}} \tilde{\beta}(\theta) d \theta} \\
t_{k} & =\frac{\Theta_{k}+\Theta_{k+1}}{2}
\end{aligned}
$$

The Lloyd-Max algorithm finds the optimal solution iteratively by following steps

1) Initialize $\left\{\Theta_{k}\right\}$ by uniform discretization.

2) Set $t_{0}=0, t_{N}=\pi$, compute $\left\{t_{k}\right\}$ using (6) for $1 \leq$ $k \leq N-1$.

3) Compute $\left\{\Theta_{k}\right\}$ using (5) for $2 \leq k \leq N-1$.

4) Repeat steps 2) and 3) until further improvement of MSE is negligible.

As shown in Fig. 2(a), we assume that light beam is transmitted along the $Z$ axis. We solve the $2 \mathrm{D}$ steady state RTE on YOZ plane. The angular direction $\vec{n}$ can be expressed in cartesian coordinates with $\vec{e}_{y} \cdot \vec{n}=\sin \theta$ and $\vec{e}_{z} \cdot \vec{n}=\cos \theta$, where $\vec{e}_{y}$ and $\vec{e}_{z}$ are the unit vectors along the $Y$ and $Z$ axis. After replacing the integral term by the summation of discrete form of phase function, $w_{k, k^{\prime}}$, the RTE on a particular discrete direction $k$ can be given as 


$$
\begin{aligned}
& \sin \Theta_{k} \frac{\partial L_{k}(\vec{r})}{\partial y}+\cos \Theta_{k} \frac{\partial L_{k}(\vec{r})}{\partial z} \\
= & -c L_{k}(\vec{r})+b \sum_{k^{\prime}=1}^{K} w_{k, k^{\prime}} L_{k^{\prime}}(\vec{r})+E_{k}(\vec{r}) .
\end{aligned}
$$

We apply the modified Simpson's rule [1] which is a method for numerical integration to compute $w_{k, k^{\prime}}$. Firstly, we compute $w_{1, k^{\prime}}$ as

$$
\begin{aligned}
& w_{1, k^{\prime}}= \\
& \begin{cases}\frac{\Delta \theta_{1}\left(2 \tilde{\beta}\left(\Theta_{1}\right)+\tilde{\beta}\left(\Theta_{2}\right)\right)}{3}, & k^{\prime}=1 \\
\frac{\left(\Delta \theta_{k^{\prime}}+\Delta \theta_{k^{\prime}-1}\right)\left(\tilde{\beta}\left(\Theta_{k^{\prime}-1}\right)+4 \tilde{\beta}\left(\Theta_{k^{\prime}}\right)+\tilde{\beta}\left(\Theta_{k^{\prime}+1}\right)\right)}{12}, & k^{\prime}=2,3, \ldots, \frac{K}{2} \\
\frac{\Delta \theta_{K / 2}\left(2 \tilde{\beta}\left(\Theta_{K / 2+1}\right)+\tilde{\beta}\left(\Theta_{K / 2}\right)\right)}{3}, & k^{\prime}=\frac{K}{2}+1 \\
w_{1, K+2-k^{\prime}}, & k^{\prime}=\frac{K}{2}+2, \ldots, K,\end{cases}
\end{aligned}
$$

where $\Delta \theta_{k^{\prime}}$ is the angle interval between discrete angular direction $k^{\prime}$ and $k^{\prime}+1$. Note that the scattering phase function is a probability density function (PDF) which means that the integration of it is one. To preserve this statistical property, we normalize the weight $w_{1, k^{\prime}}$ by $\sum_{k^{\prime}=1}^{K} w_{1, k^{\prime}}$ so that the summation of each row of the weight matrix remains one. Since the phase function only depends on the angle between two directions, we can derive all $w_{k, k^{\prime}}$ simply from $w_{1, k^{\prime}}$ as follows

$$
w_{k, k^{\prime}}=w_{1,\left|k^{\prime}-k\right|+1} \text {. }
$$

\section{B. Finite Difference Spatial Discretization}

As shown in Fig. 2(a), the highly collimated light source is placed in the middle of the left boundary and the receiver is aligned on the opposite side $X O Y$ plane. We assume vacuum boundary condition which means that the incoming radiance on the boundary of the interested area of water body is zero.

A rectangular spatial mesh is applied with $I$ grid points on the $Y$ coordinate and $J$ grid points on the $Z$ coordinate. The step sizes along $Y$ axis and $Z$ axis are $\Delta y$ and $\Delta z$, respectively. The smaller the step size is, the more accurate results can be obtained. However, there is trade-off between accuracy and computational time. Let $L_{k, i, j}$ represent the radiance at the grid point $(i, j)$ towards angular direction $k$. We replace the spatial derivatives in (7) with the upwind type finite difference. Each spatial derivative has two distinct difference formulas according to the sign of $\sin \Theta_{k}$ and $\cos \Theta_{k}$ [10]. Letting $\eta_{k}=\sin \Theta_{k}$ and $\xi_{k}=\cos \Theta_{k}$, we have

$$
\begin{aligned}
& \frac{\partial L_{k, i, j}}{\partial y} \approx \begin{cases}\frac{L_{k, i, j}-L_{k, i-1, j}}{\Delta y}, & \eta_{k}>0 \\
\frac{L_{k, i+1, j}-L_{k, i, j}}{\Delta y}, & \eta_{k}<0 .\end{cases} \\
& \frac{\partial L_{k, i, j}}{\partial z} \approx \begin{cases}\frac{L_{k, i, j}-L_{k, i, j-1}}{\Delta z}, & \xi_{k}>0 \\
\frac{L_{k, i, j+1}-L_{k, i, j}}{\Delta z}, & \xi_{k}<0 .\end{cases}
\end{aligned}
$$

For $\eta_{k}>0$ and $\xi_{k}>0$, substituting (10) and (11) into (7), we get the fully discretized form of RTE as

$$
\begin{aligned}
& \eta_{k} \frac{L_{k, i, j}-L_{k, i-1, j}}{\Delta y}+\xi_{k} \frac{L_{k, i, j}-L_{k, i, j-1}}{\Delta z}+c L_{k, i, j} \\
& =b \sum_{k^{\prime}=1}^{K} w_{k, k^{\prime}} L_{k^{\prime}, i, j}+E_{k, i, j} .
\end{aligned}
$$

For the other three cases, the procedure is just straightforward and we will not go into detail hereafter.

\section{Gauss-Seidel Iterative Method}

We apply the Gauss-Seidel iteration [11] to solve the fully discretized system of linear equations. This iteration accelerates the convergence by guaranteeing that all the values are updated by newly calculated results without delay. After some basic manipulation on (12), we get the following iterative formula

$$
L_{k, i, j}^{l+1}=\frac{\frac{\eta_{k}}{\Delta y} L_{k, i-1, j}^{l+1}+\frac{\xi_{k}}{\Delta z} L_{k, i, j-1}^{l+1}+b \sum_{k^{\prime}=1}^{K} w_{k, k^{\prime}} L_{k^{\prime}, i, j}^{l}+E_{k, i, j}}{\frac{\eta_{k}}{\Delta y}+\frac{\xi_{k}}{\Delta z}+c},
$$

where $l$ is the iterative index. The iteration process is repeated until the relative error norm $\frac{\left\|L_{k, i, j}^{l+1}-L_{k, i, j}^{l}\right\|}{\left\|L_{k, i, j}^{l+1}\right\|}$ at the grid point $(i, j)$ is smaller than a predetermined termination value $\sigma$.

\section{Computation of the Received Power}

As shown in Fig. 2, the receiver is fixed on the plane perpendicular to $Z$ axis (e.g., $X O Y$ plane). Since the radiance calculation in section II is processed on $2 \mathrm{D}$ rectangular mesh on $Y O Z$ plane, we finally get the radiance at the grid points along $Y$ axis at the receiver. In this section, we show the proposed scheme to compute the received power from the radiance calculated by the $2 \mathrm{D}$ steady state RTE. As shown in Fig. 2(b), $A_{1}, A_{2}, \ldots, A_{M}$ are the areas of the regions with the grid points located in its middle and marked with different colours respectively, which can be calculated as

$$
A_{m}=\left\{\begin{array}{l}
\pi\left(\frac{\Delta y}{2}\right)^{2}, m=1 \\
\pi\left(\frac{\Delta y}{2}+(m-1) \Delta y\right)^{2}-\pi\left(\frac{\Delta y}{2}+(m-2) \Delta y\right)^{2}, \\
m=2,3, \ldots, M,
\end{array}\right.
$$

where $M$ is the number of regions within the receiver aperture. Let $R$ be the radius of the receiver aperture, we have $M=R / \Delta y$. We assume that the scattering is symmetric in the azimuthal direction, which means that the radiance for any azimuthal angle on the same circle is the same. As such the radiance at each grid point along $Y$ axis can be regarded as the average radiance in the corresponding region where it located. Therefore, the received power can be obtained by

$$
P_{r}=\sum_{m=1}^{M} A_{m}\left(\sum_{k=1}^{K^{\prime}} \Delta \theta_{k} L_{m+\left(\frac{I-1}{2}\right), J, k}\right)
$$

where $K^{\prime}$ is the number of discrete directions within the receiver field of view (FOV).

\section{Simulation Results}

The parameters of three water types investigated in the simulation are listed in Table I. All of them are the typical values of coastal and harbor waters. The single scattering albedo is defined as the ratio of $b / c$. The step size $\Delta y=0.01 \mathrm{~m}$, $\Delta z=0.05 \mathrm{~m}$, and iterative termination value $\sigma=10^{-4}$ are 


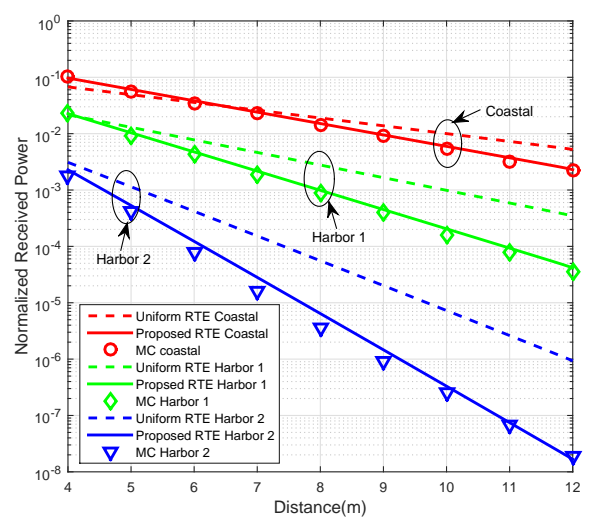

Fig. 3. Normalized received power versus transmit distance for receiver aperture $=0.1 \mathrm{~m}$ and $\mathrm{FOV}=180^{\circ}$. The number of angular directions $K=14$ is used for both proposed and uniform RTE solver.

TABLE I

PARAMETERS OF THREE WATER TYPES

\begin{tabular}{|c|c|c|}
\hline Water type & $c\left(m^{-1}\right)$ & Albedo \\
\hline Coastal & 0.568 & 0.60 \\
\hline Harbor 1 & 1.1 & 0.85 \\
\hline Harbor 2 & 2.19 & 0.85 \\
\hline
\end{tabular}

used for the proposed and uniform RTE solver. At the receiver, the receiver aperture $=0.1 \mathrm{~m}$ and $\mathrm{FOV}=180^{\circ}$ are used in the simulation. MC simulation provided in [12] is applied here for accuracy and efficiency validation of the proposed scheme. The MATLAB code of the proposed RTE solver is not included in this letter due to space limitation but it is available in its companion technical report [13].

Fig. 3 shows the normalized received power of three different schemes, i.e., proposed, uniform, and MC solvers at different transmit distance for various water types. As shown in Fig.3, while we use $K=14$, the proposed RTE slover already achieves comparable accuracy to MC simulation. However, with the same number of angular directions, there are gaps between the results from uniform RTE solver and MC simulation. We find that the uniform scheme achieves the same accuracy when $K=32$. We also note that the received power drops significantly for turbid water due to the high attenuation.

Fig. 4 shows the time gain of both the proposed and the uniform RTE solver with the same accuracy in terms of the ratio of their simulation time to that of MC. The proposed scheme is much faster than MC simulation and the former can provide results in a few seconds. Since the uniform RTE solver requires larger number of angular directions $(K=32)$, its time gain is less than the proposed scheme. Also from Fig. 4 , we can see the time gain becomes more prominent as the turbidity of the water increases. This is because the proposed RTE solver is not sensitive to the $c$ value. Changes in $c$ barely affects the simulation time since we do not change the step size. However, the MC simulation is highly affected by $c$ value since the photons encounter more scattering with higher $c$.

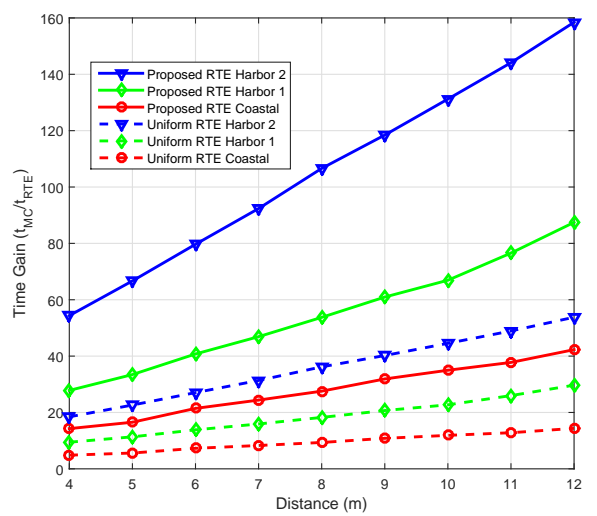

Fig. 4. Time gain of proposed and uniform RTE solver under the same accuracy ( $K=14$ and 32 for proposed and uniform scheme respectively).

\section{CONClusion}

In this letter, an efficient numerical solver was proposed based on [1] to find the steady state RTE solution. From the resulting radiance obtained from the RTE slover, we can calculate the path loss of UOWC channel at the receiver. Simulation results show that the proposed scheme can compute the received power with much faster speed than that of MC, while keeping a comparable accuracy.

\section{REFERENCES}

[1] H. Gao and H. Zhao, "A fast-forward solver of radiative transfer equation," Transport Theory and Statistical Physics, vol. 38, no. 3, pp. 149-192, May 2009.

[2] L. Lanbo, Z. Shengli, and C. Jun-Hong, "Prospects and problems of wireless communication for underwater sensor networks," Wiley Wireless Commun. and Mobile Computing, vol. 8, no. 8, pp. 977-994, Oct. 2008.

[3] J. Smart, "Underwater optical communications systems. Part 1: Variability of water optical parameters," in Proc. IEEE Military Communications Conference (MILCOM'2005), Atlantic, NJ, Oct. 2005, pp. 1140-1146.

[4] S. Arnon, J. Barry, G. Karagiannidis, R. Schober, and M. Uysal, Advanced Opt. Wireless Commun. Systems. Cambridge University Press, 2012.

[5] C. Mobley, E. Boss, and C. Roesler, "Ocean optics web book," 2010. [Online]. Available: http://www.oceanopticsbook.info/

[6] C. Gabriel, M.-A. Khalighi, S. Bourennane, P. Léon, and V. Rigaud, "Monte-Carlo-based channel characterization for underwater optical communication systems," Journal of Optical Communications and Networking, vol. 5, no. 1, pp. 1-12, Jan. 2013.

[7] C. D. Mobley, B. Gentili, H. R. Gordon, Z. Jin, G. W. Kattawar, A. Morel, P. Reinersman, K. Stamnes, and R. H. Stavn, "Comparison of numerical models for computing underwater light fields," Applied Optics, vol. 32, no. 36, pp. 7484-7504, Dec. 1993.

[8] L. Johnson, R. Green, and M. Leeson, "A survey of channel models for underwater optical wireless communication," in Proc. IEEE 2nd International Workshop on Optical Wireless Communications (IWOW'2013), Newcastle, England, Oct. 2013, pp. 1-5.

[9] S. Lloyd, "Least squares quantization in PCM," IEEE Trans. Inf. Theory, vol. 28, no. 2, pp. 129-137, Mar. 1982.

[10] A. D. Klose and A. H. Hielscher, "Iterative reconstruction scheme for optical tomography based on the equation of radiative transfer," Medical physics, vol. 26, no. 8, pp. 1698-1707, 1999.

[11] W. F. Ames, Numerical methods for partial differential equations. Academic press, 2014.

[12] W. C. Cox Jr, Simulation, Modeling, and Design of Underwater Optical Communication Systems. North Carolina State University, 2012. [Online]. Available: http://www.github.com/gallamine/photonator

[13] C. Li, K.-H. Park, and M.-S. Alouini, "A direct radiative transfer equation solver for path loss calculation of underwater optical wireless channels," KAUST Technical Report. [Online]. Available: http://hdl.handle.net/10754/333892 